\title{
COSMIC STRING WAKES AND LARGE-SCALE STRUCTURE
}

\author{
A. Stebbins $\star$, S. Veeraraghavan $\diamond$, R. Brandenberger ${ }^{\diamond}$, J. Silk ${ }^{\diamond}$, N. Turok ${ }^{\star}$ \\ * NASA/Fermilab Astrophysics Center \\ $\diamond$ Astronomy Department, University of California, Berkeley \\ Physics Department, Brown University
}

Cosmic Strings are one-dimensional topological defects that may be formed in the early universe during a phase transition, and which may be the source of all inhomogeneities in our universe. Their mass per unit length, $\mu$, gives us a dimensionless parameter, $\mu_{6} \equiv 10^{6} G \mu / c^{2}$, which must be of order unity for strings to seed galaxy formation. Results to date from the ongoing CfA redshift survey ${ }^{1}$ suggest that galaxies are distributed on two-dimensional surfaces, whose typical separation is about $50 h_{50}^{-1} \mathrm{Mpc}$. The loop distribution is unlikely to imprint such large-scale patterns in the galaxy positions so we have examined whether this structure could be caused by infinite strings. Because an infinite string typically moves at a substantial fraction of the speed of light, it will leave behind a very large accretion wake in the ambient medium. Gravitational instablity causes these wakes to continue to accrete matter long after the string has moved elsewhere. These wakes form around the two-dimensional surfaces swept out by the long strings.

We have studied whether these accretion wakes could be associated with the observed sheets of galaxies ${ }^{2}$. Only in a nearly flat universe $\left(\Omega_{0} \approx 1\right)$ containing cold dissipationless matter are the present day wakes comparable in surface density to the observed sheets of galaxies. The 'most prominent' wakes (i.e. those with the largest surface density) were formed by strings around the era of matter-radiation equality. The present thickness and surface density of the nonlinear regions of these prominant wakes is $\sim 3 \mu_{6} \mathrm{Mpc}$, and $4 \times 10^{11} \mu_{6} h_{50}^{2} M_{\odot} / \mathrm{Mpc}^{2}$ respectively. Given published estimates of the density of long strings ${ }^{3}$, the typical seperation of the prominant wakes at the present epoch should be $\sim 40 h_{50}^{-2} \mathrm{Mpc}$. Thus these wake structures compare very favorably with the observed sheets of galaxies.

However several difficulties remain. Wakes do not suppress the collapse of matter onto loops nor are they able to accrete most loop-seeded objects onto themselves. The galaxy-sized loop-seeded objects are found to contain more matter than the wakes and these objects will remain in the regions between the wakes. Thus one would have to invoke some sort of environmental biasing to prevent the loop-seeded objects from becoming observable galaxies if one wished to interpret the observed sheets as being the cosmic string wakes. Furthermore preliminary indications from recent numerical simulations suggest that the density of long strings is appreciably larger than previously thought ${ }^{4}$ which may indicate that the typical inter-wake distances are smaller than the typical void size. While final conclusions must await completion of these simulations it seems unlikely that cosmic string wakes explain the large-scale structure.

\section{References}

1 V. de Lapparent, M. Geller, \& J. Huchra 1986, Ap. J. Lett. 302 L1.

2 A. Stebbins, S. Veeraraghavan, R. Brandenberger, J. Silk, \& N. Turok 1987, Ap. J. 322, 1.

3 A. Albrecht \& N. Turok 1984, Phys. Rev. Lett. 54, 1868.

4 D. Bennett private communications; N. Turok private communications. 562

J. Audouze et al. (eds.), Large Scale Structures of the Universe, 562.

(c) 1988 by the IAU. 